CORRECTION

https://doi.org/10.1038/s41586-019-1221-4

\title{
Author Correction: A dissipatively stabilized Mott insulator of photons
}

Ruichao Ma, Brendan Saxberg, Clai Owens, Nelson Leung, Yao Lu, Jonathan Simon \& David I. Schuster

Correction to: Nature https://www.nature.com/articles/s41586-0190897-9, published online 06 February 2019.

Two references ${ }^{1,2}$ were inadvertently omitted from this Article. They have been added as refs ${ }^{12}$ and ${ }^{14}$ at the end of the sentence "Recently, photonic systems have emerged as a platform of interest for the exploration of synthetic quantum matter." in the original Article. Subsequent references are renumbered accordingly.

1. Hartmann, M. J., Brandão, F. G. S. L. \& Plenio, M. B. Strongly interacting polaritons in coupled arrays of cavities. Nat. Phys. 2, 849-855 (2006).

2. Angelakis, D. G., Santos, M. F. \& Bose, S. Photon blockade induced Mott transitions and $X Y$ spin models in coupled cavity arrays. Phys. Rev. A 76, 031805 (2007). 